\title{
Sampling Operation with Robotic UAV
}

\author{
Edmundo Guerra, Antoni Grau, Yolanda Bolea \\ Automatic Control Department \\ (ESAII) \\ Technical University of Catalonia (UPC) \\ Barcelona, Spain \\ orcid.org/0000-0002-6696-0982, antoni.grau(at)upc.edu
}

\author{
Rodrigo Munguia \\ Department of Computer Science \\ (CUCEI) \\ University of Guadalajara, \\ Guadalajara, Mexico \\ rodrigo.munguia(at)upc.edu
}

\begin{abstract}
This work presents a solution to automatize sampling tasks in a wastewater treatment plant with open air basins. At the behest of human operators, a set of UAVs managed as a network of autonomous agents will perform sample missions by taking direct measurements (through a multiparametric probe) or capturing samples and carrying them to the laboratory with specific developed hardware. These capabilities allow the proposed solution to act as a virtual sensor network with sampling points deployed and connected at any point reachable by UAVs. The hardware prototypes are fully described, with focus on the integration of systems, and the software architecture used is analysed and fully justified. Special focus was put on the localization problem, and several solutions were evaluated. Experimental results of the prototype UAV and sampling probe built are provided to validate the hardware designs, with focus on the localizations tasks.
\end{abstract}

Keywords-Unmanned aerial vehicle, robotic software architecture, specific tools, robotic agents

\section{INTRODUCTION}

Monitoring and surveying require sampling of products several times, for instance in wastewater treatment plants. In a typical treatment plant, waters with different levels of pollution need to be tested frequently, following concrete specifications and normative, sampling different basins and tanks. These operations require capturing samples from different points in-site, being a task that is cumbersome, frequent, and systematic, thus being the kind of procedure that could be automated. Given the open structure of an outdoor wastewater treatment plant, with open air tanks and basins, UAV could hypothetically reach most of the sampling points, with better a ccessibility than human operators. Then, being a known open environment, the navigation challenges and risks can be managed with current technologies.

In order to automatize these operations, the robotic UAVs need to have enough autonomy of operation to act on their own agency: they will have to take decisions with respect to mostly known tasks and environments that cannot be completely modelled and represented. There are several design approaches and technologies when trying to produce robots with these capabilities, being one of the most fruitful the multi-agent system (MAS). Under this paradigm, the different tasks or parts of the problem to solve are distributed to different agents, which focus on their own parts. This simplifies each agent (as it only solves one task or problem), who only needs knowledge about its task (easing data processing an management operations), and who only depends on a small number of other agents, if any (isolating processes and execution threads). A good example of how to exploit all these features in a robotics MAS can be found in [1], where based on MAS methodologies [2], a solution for a surveying surface vehicle is implemented over the ROS framework [3].
Authors earlier works [4], presented an architecture for a single unmanned aerial vehicle working jointly with a unmanned surface vehicle in a wastewater plant. Loosely based on that work, a new proposed system supports a network of UAV platforms, which can act as a high density virtual sensor network. This works describes the sampling problem and the general view of the solution proposed, followed by a detailed hardware description with focus on the integration. The next section describes the software design approach taken, based in the MAS architecture. To conclude, experimental results in terms of the accuracy of the localization of the UAV and the design of the sampling probe are provided, and some discussion on the results achieved and future works.

\section{UAV NETWORK SAMPLING OF WASTEWATER PLANTS}

In terms of management, one of the most critical processes to properly monitor a wastewater treatment plant is the evaluation of processed water, to ensure its compliance with the required specifications. The factors to be accounted and monitored include from chemical properties of the water, like $\mathrm{pH}$, to concentrations of biological and inorganic pollutants. This frequently means obtaining multiple samples, with variable specifications [5]. For tests with periodic testing requirements this means planning account for both the requisites and the resources available, be it technical or human.

For most of the metrics to test, both for clean waters and wastewaters, one of the most desired features is the homogeneity of the water to be sampled. This guarantees that the results obtained are representative of the water present in a given basin or tank, providing validity to the analyses. Notice that process tanks in water treatment plants present slow dynamics, with low flow velocities that drag dilution processes along all the tank. This fact produces hard to model delays, where water subjected to different rates of treatment can be present concurrently in the tank. This means that proper representative sampling of a single basin may require several samples at different points.

To address these challenges, the proposed system offers a solution for the sampling and analyses required to monitor and supervise the open air basins in a wastewater treatment plant. The sampling network allows to analyse the waters present in almost every point of the open basins and tanks, thus, acting as a virtual network of multiparametric sensors with hundreds of probes deployed. To achieve this, the robotic UAV platforms are fitted with the necessary probing equipment: either a sample collector probe which picks water samples around $\sim 0.41$ to bring them to a delivery station; or a multiparametric probe sensor to sample the available parameters online while in flight. 


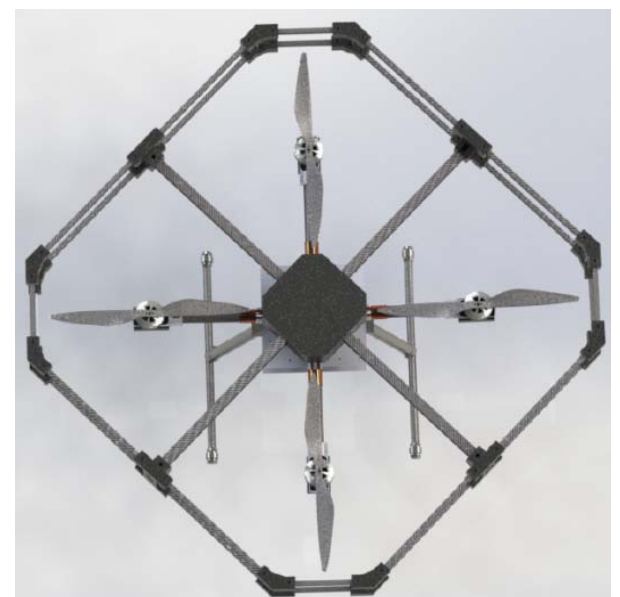

Fig. 1. Render of the prototype UAV platform design developed to build the sample collector UAV part of the UAV sensing network.

Thus, a human controller operating the system is able to set a list of sampling tasks. Each one of this tasks is composed of the coordinates of a point in one of the basin, where the sampling is to take place, and a set of instruction or restrictions related to the sampling, including which parameters are desired to measure, which time or period should the measurements or samples to be taken, whether is required to keep the sample or a single measurement is enough, and any other features.

When there are conditions that makes the use of a multiparametric probe unsuitable (measurements that require hours or day so that the oxygen is consumed, the normative requires a contrast analysis with the same sample, etc...), a similar UAV with a sample collector probe (described in the results section) will be sent. The operations of these UAVs are more complex, as the deploy a probe that can be empty or filled with water, modifying the dynamic behaviour of the UAV. To fill it, the UAV will submerge the probe into the air basin by floating above the water, as the sample collection probe is rigidly solidary with the UAV. After enough time to fill the probe has passed, the UAV flies to a delivery station, where it releases the magnetic valve which sustains the probe.

\section{SYSTEM AND HARDWARE STRUCTURE}

The system proposed is largely based in off-the-shelf (OTS) commercially available technologies for hardware, and open source software. The system is managed from a central control system, which is deployed in an ordinary PC, thus allowing to manage a network of sample collector UAVs and localization beacons.

The sample collector UAV was designed as a quadcopter at X4 configuration, due its stability and performance, with a length of $0.664 \mathrm{~m}$ between rotor axes (see Fig. 1). The propellers are standard 18' build in carbon fiber, with TMotor MN4014 actuators. These rotors are controlled at low level by a set of 4 T-Motor AIR 40A electronic speed controllers (ESC), which are connected to a standard power supply board paired to a Pixhawk 2.4.8 flight management unit (FMU). The power supply board is connected to a custom power system, feed by two $6 \mathrm{~S} 10,000 \mathrm{mAh}$ batteries.

The Pixhawk FMU is connected to a EGNOS SBAS enabled Ublox GPS, an internal IMU, a Lightware SFC10/B LRF set as altimeter, LRF two radio transceivers: a $915 \mathrm{Mhz}$ radio telemetry module; and a Futaba 2008RB 2.4Ghz to enable direct manual control during experimental validation of newly developed features. To run all the sensors and hardware the Pixhawk is set with the PX4 flight stack, providing all the basic flight capabilities. This quadcopter setup has a maximum take-off weight of $13.9 \mathrm{~kg}$, with a payload weight ranging between 3.9 and $4.55 \mathrm{~kg}$ depending on the presence and configuration of a safety cage.

This base UAV was enhanced with robotic capabilities after the addition of several sensors and an Odroid X4 single board computer (SBC). This SBC supported several devices, including: a USB 3.0 camera (up to 5MP, usually set a VGA resolution to ease bandwidth usage during monitoring), a SBAS (EGNOS network) receiver, a network beacon transceiver, and a set of 4 MaxBotix ultrasonic sensors to detect near-collisions and obstacles. The final addition to the UAV system is the sampling system, with two different options: deployment of a multiparametric probe or the deployment of a custom sample collector probe, described at a later section. A full schema of the computing architecture and all the devices connected can be seen in Fig. 2.

It is useful to consider the distributed sampling network developed in terms of a set of different subsystems, with each subsystem comprised of both the hardware and software components responsible for a task. The hardware level components are generally organized according physical restrictions and specifications, while at software level, a multiagent system (MAS) approach, based on the methodology described in [1], was considered the most convenient approach. Using a MAS design abstraction allows produce software level components with great modularity and robustness, which are integrated with the hardware level components through a set of low-level routines and drivers, which build an interface layer. In terms of software implementation, the multi-agent architecture uses a framework based on ROS middleware.

Notice that several systems will be present in each instance of the sampling network, i.e., for each of the UAV deployed; while other will work akin a singleton class, with only one instance for the whole network sampling system. The subsystems present once are those related with coordination, management and infrastructure:

- Main Control subsystem: This subsystem is composed of a set of ground stations to supervise the avionics of the UAVs and a control and supervision station for the sampling network.

- Beacon network subsystem: It is composed of a beacon sensor network used to help with UAV localization.

- Communications subsystem: The communications are implemented using multiple wireless channel. The radio telemetry from each of the FMU is observable through a ground station, while a ROS network is run over $4 \mathrm{G}$ through a VPN based on OpenVPN, enabling full communication through ROS.

On the other hand, the prototype sample collector UAV presents its own set of subsystems, once per UAV:

- Robotics Control subsystem: This subsystem is composed of the Odroid XU4 SBC and the sensors and devices connected to it, providing the autonomous capabilities.

- Avionics subsystem: It is composed of all the hardware and software required for basic flight operations, 
including a PixHawk FME, ESCs, motors, GPS sensor, and landing gear controller and servos.

- Power subsystem: This system provides power to all the devices on-board a UAV, including the avionics and robotics subsystems, with a custom board to balance batteries and produce several voltajes.

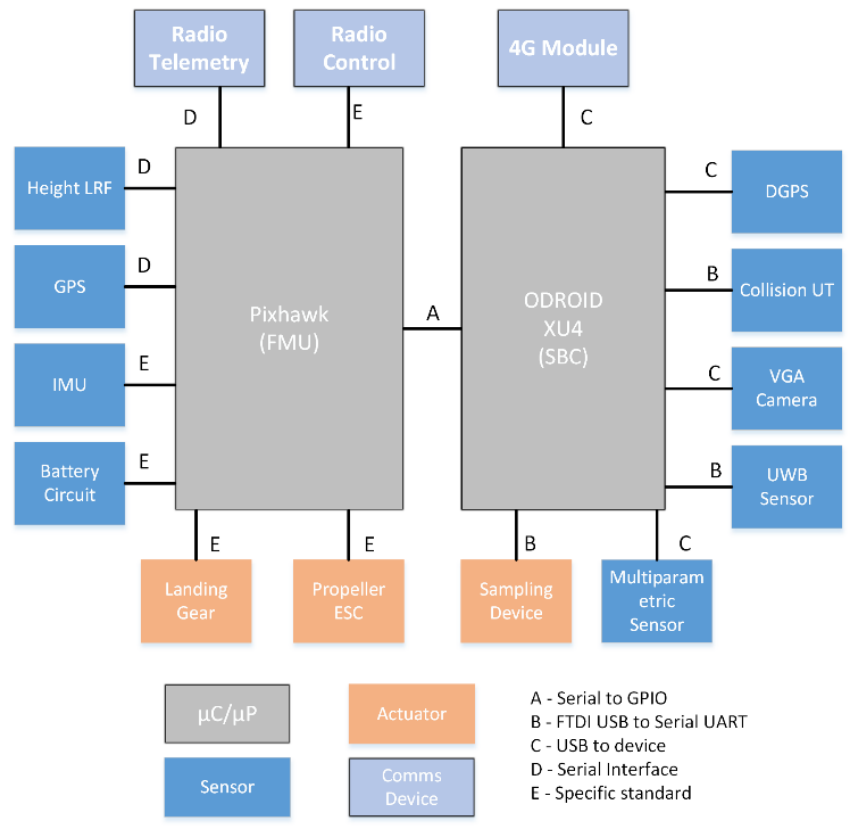

Fig. 2. Hardware architecture of the prototype sample collector UAV developed. Due the weight and size limits, only the sampling device or the multiparametric sensor are deployed concurrentlyThe DGPS (Differential GPS) has been only available for validatoin tests.

\section{System Design AND SOFTWARE ARCHITECTURE}

The different software components used in the proposed system can be described as a set of Agents which are organized according to a multi-agent system (MAS) architecture, which is largely implemented over a ROS framework [3] .This Multi-agent system is a convenient abstraction, providing a virtual infrastructure to organize and design the high level software capabilities. The ROS framework is run in an ODROID SBC deployed at the sample collector UAV, and the PC machine/s present at the main control subsystem. The flight management unit also runs several pieces of software at agent level, those closely related with the avionics systems of the UAV, and usually closely tied to the low level control routines communicating with the hardware level.

Note that a ROS framework usually requires that a single "ROS master node" (being a node the basic execution unit that communicates with other nodes through ROS) acts as a "master agent" managing all the routing and initial steps of every communication. This centralized architecture presents a critical fail point in this feature, as failure of this "node" usually invalidates the whole network. In order to avoid the commented issues related to a master "node" acting as master agent, several master agents will be deployed using the multimaster_FKIE tool, reviewed at [6].

Thus, the agent level software used in the system can be grouped based on the execution environment. Firstly, we have those agents running in the PC stations of the main control subsystem:
- Central Master Agent

- User Agent

- Sampling network manager

- Central Logger Agent

- Beacon network Agent

Secondly, we have those agents related with the robotics control subsystem, or closely related to it, which are executed in the SBC:

- Local Master Agent:

- Mission Management Agent

- Navigation Agent

- Localization Agent

- Camera Agent

- UT Collision Agent

- DGPS Agent

- Beacon network sensor Agent

- Multiparametric Sensor Agent

- Sampling Device Agent

- Local Logger Agent

The last group of agents is executed in the FMU, thus are parts of the flight stack PX4. Notice that their description as agents is mainly virtual, as entities with specific tasks, resources, and their own agencies and procedures defined, which although they are part of the same software stack, present their own interfaces in the ROS framework under the guise of their respective topics and services:

- Avionics Agent

- Power management agent

- Low level control agent

- Landing Gear Agent

So, even being only virtual agents, from a design point of view they are part from the multi-agent system, and will complete the MAS architecture. Notice that even the agents that are implemented in the FMU, so not coded as conventional "nodes", present the same communication capabilities in the ROS framework as they interface through the MavROS library.

\section{EXPERIMENTAL RESULTS}

\section{A. Water Sampling with an UAV}

The hardware design and software architecture described in previous sections consider two different available setup options to sample the open air basins: using a commercial OTS multiparametric probe sensor or deploying a custom designed sample collection device, able to hold over $350 \mathrm{~cm} 3$. The first option presents two main advantages: it is an existing technology, widely supported, already integrated into PC environments, and from a flight and control point of view, a hanging solid body with fixed mass is a problem already solved by research in with several strategies. On the other hand, deploying such hardware would be financially expensive.

The second option also presents its own weaknesses and strengths: deployment of a hardware system able to collect water samples can be cheap and be used in processes where the sample must be kept, cheap but it cannot sample on the fly. Note that the utilization of the sample collector probe implies that the mass of the UAV platform (so the inertia tensor) will vary dynamically, disturbing low level control loops. 


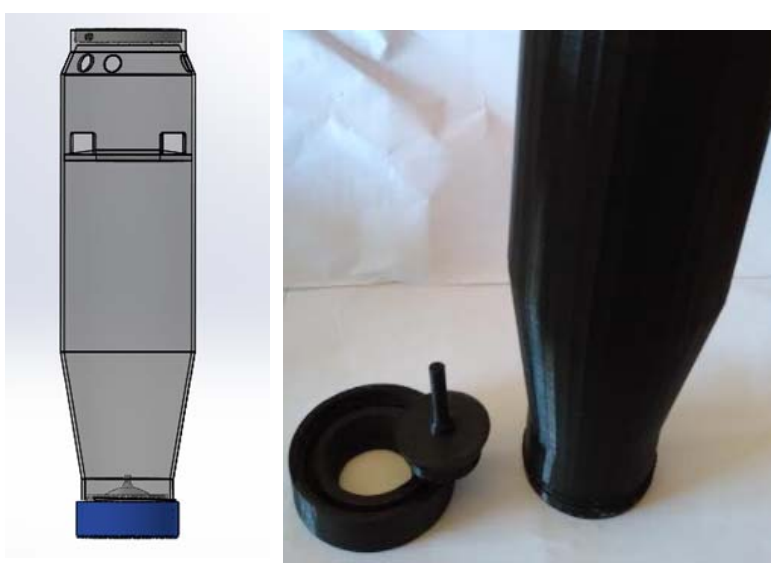

Fig. 3. Details of the designed sample collector probe. Left side: CAD design of the probe, with details of the buoyancy valve. Right side: $3 \mathrm{D}$ printing prototype of the probe.

The proposed sample collection probe was developed and built, see Fig 4 . The probe is able to hold $\sim 0.41$ according to the fill tests, with a buoyancy valve that will lock the containment part as it floats to its top when filled. The probe is attached to the UAV by a ferromagnetic plate at its top, which is locked into a magnetic gripper. The probe has to be submerged for $3 / 4$ of its height, so that waters reach the opposing entry holes, whose position ensure that the flow velocities of entering water induce negligible torque.

TABLE I. TABLE TyPe Styles

\begin{tabular}{|c|c|c|c|}
\hline \multirow{2}{*}{ Measurement } & $\begin{array}{c}\text { Nominal } \\
\text { errors }\end{array}$ & \multicolumn{2}{|c|}{ Experimental error $^{\mathbf{a}}$} \\
\cline { 3 - 4 } & $\boldsymbol{A v g .}$ & $\boldsymbol{\sigma}^{\mathbf{2}}$ \\
\hline $\begin{array}{c}\text { GPS only } \\
\text { (instantaneous) }\end{array}$ & $\begin{array}{c}13 \mathrm{~m} \text { (planar), } \\
22 \mathrm{~m} \text { (height) }\end{array}$ & $\begin{array}{c}6.9 \mathrm{~m} \text { (planar), } \\
9.2 \text { (height) }\end{array}$ & $\begin{array}{c}3.2 \mathrm{~m} \text { (plane), } \\
\text { (height) }\end{array}$ \\
\hline $\begin{array}{c}\text { GPS with } \\
\text { EGNOS (instant) }\end{array}$ & $\begin{array}{c}3 \mathrm{~m}, 8 \mathrm{~m} \\
\text { (height) }\end{array}$ & $1.3 \mathrm{~m}$ & $0.4 \mathrm{~m}$ \\
\hline $\begin{array}{c}\text { Avionics Agent } \\
\text { (with EGNOS) }\end{array}$ & $* \mathrm{~b}$ & $\begin{array}{c}1.1 \mathrm{~m} \text { (planar), } \\
0.034 \mathrm{~m} \\
\text { (height) }\end{array}$ & $\begin{array}{c}0.27 \mathrm{~m} \text { (plane) } \\
0.002 \mathrm{~m} \text { (alt) }\end{array}$ \\
\hline $\begin{array}{c}\text { RMCB system } \\
\text { (instant) }\end{array}$ & $* \mathrm{~b}$ & $1.7 \mathrm{~m}$ & $0.6 \mathrm{~m}$ \\
\hline
\end{tabular}

a. Compared to DGPS results as ground truth

${ }^{\text {b. }}$ No nominal error available

\section{B. UAV Localization}

For the localization tasks, serveral options have been studied. Though differential GPS can produce the most accurate results, its use requires deployment of expensive equipment both in the UAV and as a part of infrastructure, and its rate $(5 \sim 10 \mathrm{~Hz})$ is not enough. All the other GPS-based solutions, like SBAS also share this specific issue. As it can be observed in Table 1, neither of the GPS-based approaches produce an accurate enough height estimation to perform the required manoeuvres to sample the basins flying close to the surface. To solve this issue, an LRF (laser range finder) altimeter was deployed.

Though the results produced by the avionics system fuse readings from all the sensors, the availability of the EGNOS enhancement is not guaranteed. To deal with the eventuality of EGNOS shortage, a system of beacons based in the TI chip
CC2530 has been tested. This component implements a full SoC solution (system on a chip) for ZigBee-based applications. This system would be deployed around the open air basin, to improve UAVs localization during EGNOS shortages. To test it, a set of 6 beacons was deployed in a planar configuration covering an area of $30 \mathrm{~m}$ per $15 \mathrm{~m}$. These tests reported errors compatible with those reported within previous works [7]. Still, active research in the field is producing new solutions[8] that could improve the results.

\section{CONCLUSIONS}

The solution proposed presents several contributions over other alternatives and works. The system presents optimal software modularity. The system architecture is fully scalable, so new sampling UAV can be added to the sampling network system with ease. In terms of the sampling process, the designed probe has been tested in simulated tanks, and its performance meets the specifications. The design with two entry points allows to reduce possible torques to negligible values, and allows the correct filling of the sample container. The actual performance of the robotics system has been only validated in terms of localization during flights. As it is one of the hardest challenges for UAV, several options have been studied.

Future works must address some specific challenges still pending, starting with a robust flight controller able to maintain the UAV over water while the sample collector fills needs to be tested. This specific challenge presents a strong dependency with the localization problem, as the controller must account for which specifications are guaranteed by the localization agent in terms of accuracy, rate and robustness.

\section{ACKNOWLEDGEMENT}

This research has been funded by Spanish Ministery of Innovation and Science CICYT DPI2016-78957-R.

\section{REFERENCES}

G. Conte, D. Scaradozzi, D. Mannocchi, P. Raspa, L. Panebianco, and L. Screpanti, 'Development and Experimental Tests of a ROS Multi-agent Structure for Autonomous Surface Vehicles', Journal of Intelligent \& Robotic Systems, vol. 92, no. 3-4, pp. 705-718, Dec. 2018.

[2] P. Langley, J. E. Laird, and S. Rogers, 'Cognitive architectures: Research issues and challenges', Cognitive Systems Research, vol. 10, no. 2, pp. 141-160, Jun. 2009.

[3] M. Quigley et al., 'ROS: an open-source Robot Operating System', in ICRA workshop on open source software, 2009, vol. 3, p. 5.

[4] E. Guerra, Y. Bolea, A. Grau, R. Munguía, and J. Gamiz, 'A solution for robotized sampling in wastewater plants', in IECON 2016 - 42nd Annual Conference of the IEEE Industrial Electronics Society, 2016, pp. 6853-6858.

[5] 'Urban Waste Water Directive - Environment - European Commission'. [Online]. Available: ec.europa.eu/environment/water/waterurbanwaste/legislation/directive_en.htm. [Accessed: 28-Feb-2019].

[6] S. Hernandez and F. Herrero, 'Multi-master ROS systems', IRITR-15-1, 2015.

[7] Y. Guo and X. Liu, 'A Research on the Localization Technology of Wireless Sensor Networks Employing TI's CC2530 Instrument', 2015, pp. 446-449.

[8] L. Thammavong, K. Khongsomboon, T. Tiengthong, and S. Promwong, 'Zigbee wireless sensor network localization evaluation schemewith weighted centroid method', MATEC Web Conf., vol. 192, p. 02070, 2018. 